\title{
EXPERIMENTAL DISCUSSION ABOUT THE INFLUENCE OF THE PROFICIENCY IN TANKENDO IN THE FOOT POSTURE Maki Nakamura ${ }^{*}$, Kiyoshi Hoshino ${ }^{2}$ \\ ${ }^{1}$ Ibaraki Prefectural University of Health Sciences Department of Nursing, Ami 300-0394, Japan, ${ }^{2}$ University of Tsukuba Graduate School of Systems and Information Engineering, Tsukuba 305-8573, Japan. \\ Email: ${ }^{1 *}$ nakamuramk@ipu.ac.jp, ${ }^{2}$ hoshino@esys.tsukuba.ac.jp
}

Article History: Received on $18^{\text {th }}$ July 2019, Revised on $27^{\text {th }}$ August 2019, Published on $28^{\text {th }}$ September 2019

\begin{abstract}
Purpose: In martial-art plays using a weapon, the length and weight of the used weapon determine the timing of attacking the opponent and playing strategies, which may be in turn reflected on the body posture and foot posture of a player. The objective of this study is to analyze the features of the food posture of a skilled player in Tankendo, in which players play using a short and light bamboo knife-like sword in order to consider an effective playing strategy.
\end{abstract}

Methodology: In our experiment, the subjects were asked to take the foot posture at the temporal and spatial timing kept to measure and quantify kinematic feature quantities, weight distribution between both the feet placed in the front and rear, respectively and favorite jump distance of them. Then, a one-to-one correlation analysis was conducted among all the feature values.

Main Findings: The result of the analysis demonstrated that a strong negative correlation was observed between the number of years of experience in Tankendo and the favorite jump distance. Similarly, a strong negative correlation was observed between the number of years of experience in Jukendo and favorite jump distance. On the other hand, in the case of Kendo, a strong negative correlation was observed between the number of years of experience in Kendo and the width between the feet placed on the right and left sides.

Implications/Applications: A series of results obtained from the study suggests that in Tankendo and Jukendo, players have such a tendency that they first would take a position near the opponent and then jump there up to strike the opponent. In contrast, in case of Kendo focusing on a thrusting technique, such a tendency was observed that with a narrow width between both the feet, players stroke their opponent so that speedy footwork might be achieved, despite easily losing body balance.

Keywords: Tankendo, foot posture, favorite jump distance, width between right and left feet, temporal and spatial timing of attacking an opponent, correlation analysis.

\section{INTRODUCTION}

Tankendo is one of the martial-art plays, originated from Japan, which may be enjoyed by everyone and everywhere. That is because a short and light bamboo sword (Tan-chiku-to) of $53 \mathrm{~cm}$ in length and $250 \mathrm{~g}$ in weight, which may be easily handled by players regardless of their body constitution and arm force, is used as shown in Fig. 1. Moreover, in Tankendo, a player fights at a close distance from an opponent compared with that in Kendo (HoshinoLab, 2015). Standing at a relatively short distance (spatial) timing from the opponent, a player hits on or defends from the opponent using a short bamboo sword or the flat part of a sword (for a sword, the most sharpened part, the point of a sword). The body sites where hitting is effective in Tankendo are the right and left face sections, throat, and forearm as shown Fig.2.

Tankendo, which requires quick determination and motions with body balance kept is believed to be useful in health enhancement and fall-prevention of elderly persons. Taking it into consideration that many of elderly persons and women tend to have an awareness that they are not good at exercise,

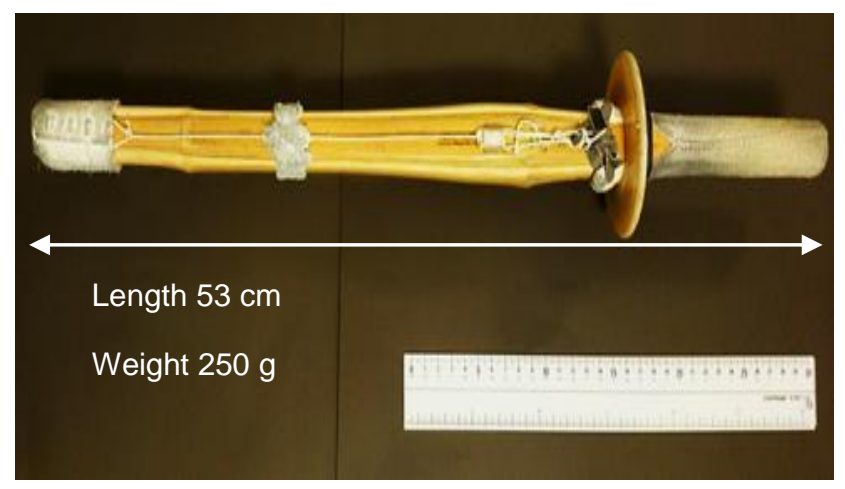

Figure 1: Short Bamboo Sword 


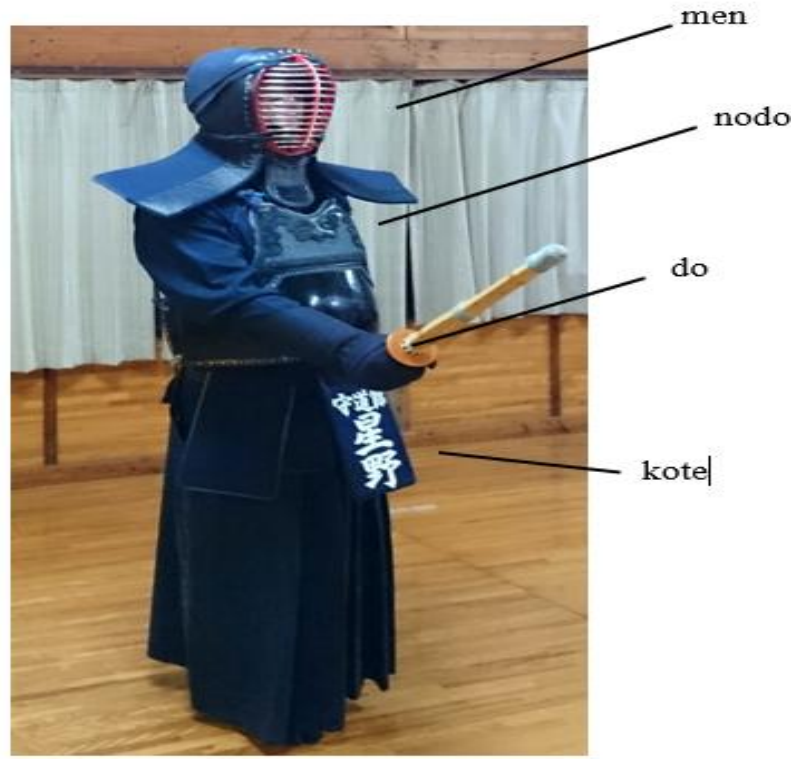

Figure 2: Areas of Valid Strikes and Thrusts

The Tankendo machine, which enables them to joyfully and safely tone up their muscles of legs, has developed (Hoshino \& Cheng, 2016; Shimanoe, \& Hoshino, 2019; Hoshino, 2018). In particular, elderly persons, who are difficult to keep body balance due to various factors such as deteriorated muscle force and visual power, may eliminate the need for nursing care if they continue this type of exercise stating from relatively young age to strengthen the muscle of legs (Northey, Cherbuin, Pumpa, Smee, \& Rattray, 2018).

In Tankendo using a short bamboo sword and Kendo using a relatively long bamboo sword, effective techniques are largely classified into two categories, striking and thrusting. The former means the motion, in which a player brings down to the opponent to cut. The later means the motion, in which player thrusts the tips of a sword or spear into the opponent. However, in Kendo using a long bamboo sword, an effective site for thrusting is only the throat but it is difficult to thrust accurately the narrow-area of the throat (http://www.kendo-progress.com/; Aloofy et al, 2017). For this reason striking techniques such as face striking, body striking and arm striking occupy the most of playing motions. In contrast, in Tankendo using a short bamboo sword, thrusting techniques occupy the most of playing motions, for example, victory or defeat was determined by means of thrusting techniques in the final round of all the games in 2016 National Tankendo Championship (HoshinoLab, 2019). As shown in Fig.3, striking techniques in Kendo include three types; 1) striking applied to the face and arm; 2) thrusting applied to the throat and body, and 3) holding the opponent's arm at the moment of approaching to each other to suppress his/her motion and thrust.

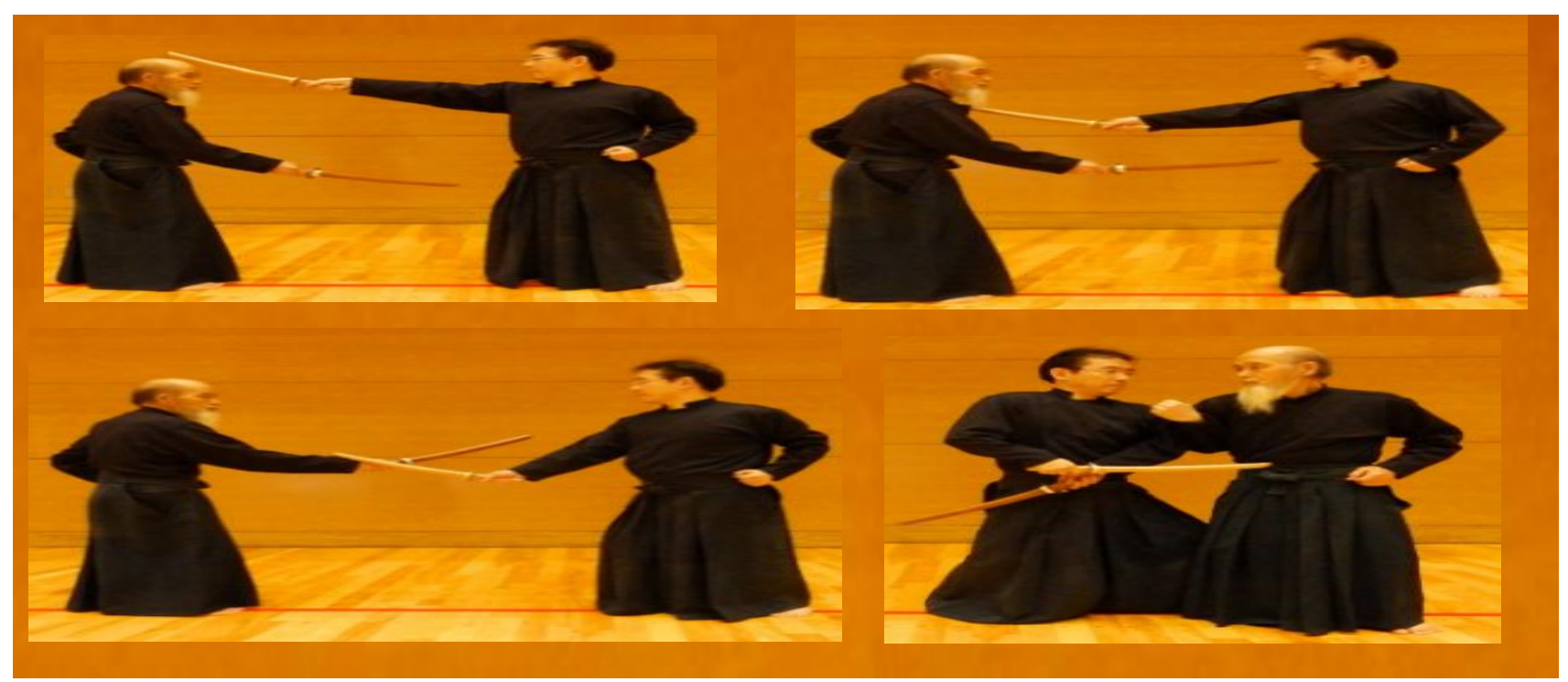

Figure 3: Techniques of Strike and Thrust 
A comparison is made among the techniques of domestic and overseas other "martial-art games using any of weapons". In Kendo mainly using striking techniques to attack the opponent, a player takes such a posture that his/her upper body faces straight to the opponent and both the feet face horizontally to the direction of movement with the right foot in the front. On the other hand, in fencing mainly using thrusting, a player turns the directions of his/her upper body and left foot by 90 degrees relative to the direction of movement. For this reason, in fencing, the relationship between the front foot, which is a dominant foot, and the rear foot, which is a pivoting foot, is likened to the hands indicating 9:00 on the clock face (Turner \& Harmenberg, 2017). However, in "sabre" among fencing events, both the striking and thrusting techniques are effective; therefore, the body posture and foot posture has taken by a player seem to be somewhat different from those of other two events. Moreover, similarly in Jukendo, another martial-art event originated from Japan, a player faces his/her upper body takes a posture almost at a right angle to the direction of movement to thrust the opponent with an oblique stance. However, in Jukendo, a player places his/her left foot in the front and his/her right foot at a distance separating horizontally on the outside so that both feet appear as if they indicated 2:00 on the clock face. In addition, in Tankendo using both the striking and thrusting techniques, some players take the foot posture similar to that in Kendo while others take like that in fencing. However, the players have to wear hakama in the games and practices in Tankendo and the foot posture of the player has not been known.

In Kendo, the words representing a lesion, "ichigan, nisoku, santan, shiriki” is used. This lesson teaches that 1) "watching the opponent's motions accurately" is the most important"; 2) "footwork" is the second important; 3) "ability to make a decision" is third important, and 4) "muscle force" is fourth important. As known from this lesson, although "hands and arms", which manipulate a weapon, and "muscle force", which drives the hands and arms, seem to gain recognition in martial-art plays, in fact, weight is laid mainly on "footwork" (http://www.geocities.jp/themusasi2g/gorin/g403.html/). During a brief moment of offense and defense in Tankendo, where an attempt is made to win an opportunity for quick striking and thrusting, a player may be required to take a foot posture such that he/she may start to move in any of eight directions, back and forth, right and left, diagonally forward right, diagonally backward right, diagonally forward left and diagonally backward left, at his/her quick discretion.

The previous study on the foot posture in Kendo (Koshida \& Matsuda, 2013; Kubo,Kizuka,Saitoh, Mutoh, Koda, \& Satoh, 2001, Tatsumi \& Hattori, 1991; reported that the position of both the feet and weight distribution are significantly related to the degree of skill at striking and thrusting and focusing on weight distribution, weight is moved toward the right foot with the opponent faced but not with no opponent faced. However, all these reports are related to Kendo focusing on striking. It may be forecast that the foot posture taken by a player determines the playing strategy and his/her special skill but the foot postures taken by a player in Tankendo using both striking and thrusting have not yet been reported.

One of methods to measure the foot posture is the use of various types of sole pressure distribution measurement instruments or footprint measurement instruments (Hoshino, Tamaki, \& Tanimoto, 2007; Hirose, 1998; Hu, Jiang, Takaki, \& Ishii, 2018; Maruyama, Tada, \& Toda, 2019; Miyajima, Tanaka, Miyata, Tada, Mochimaru, \& Izumi, 2018; Purkayastha, Byrne, \& O'Malley, 2013; Sugimura, \& Hoshino, 2017). They are capable to measure the footprint distribution of small sites such as toes accurately but have disadvantages including large-scale, expensive and not easy to measure. Most of them request the players to put their legs together during measurement. For this reason, they can be used only in narrow areas and are not suitable for measuring the foot postures with both the right and left legs separated as seen in Tankendo. A simple and safe method for measurement with no burden on the subjects includes, for example, the method of tracing the foot posture and the degree of separation of the heel from the floor on a paper, or the method of the use of two weight scales to measure the weight distribution of feet placed in front and rear. The objective of this study is to clarify the features of Tankendo as playing strategies and martial-art play by quantifying the features of foot postures in a short moment during which a player may start to move any time. Specifically, it will be experimentally shown using correlation analysis that what type of factors, such as player's skill, physical constitution and jumping distance at striking and thrusting, may determine the relationship and separation between the positions of right and left feet, and how to put the body in the center.

\section{EXPERIMENTAL}

\section{Methods}

The experimental method is described below. An A1-size paper $(594$ x $841 \mathrm{~mm})$ is attached on a flat wooden floor. First, the subjects with bare feet were requested to take his/her favorite foot posture at "a temporal and spatial timing of striking an opponent" on that paper. In front of the subject, the opponent made face the subject with a short bamboo sword. Here, a temporal and spatial timing of attacking the opponent means an inter-personal distance where a player may strike the opponent by one-stepping forward while may avoid striking by the opponent by one-stepping backward. Next, the subjects traced the outlines of their feet on the paper with a fine felt tip pen. Moreover, in order to determine whether the heels were separated from the floor, a thin clear file was used to verify the position where the clear file might be inserted into the deepest point from the three directions, right, left and back sides.

Second, the subjects removed their feet from the paper. Two weight scales were put on the positions where the feet were placed. The subjects stood on the scales with the same standing posture to measure the weights exerted on both the feet. 
Third, the subjects moved to the position slightly apart from the weight scales and paper and reproduce their foot posture. Then, they jumped over the largest one of their favorite distances seen in the game with body balance kept and the distances were recorded as shown in Fig. 4.

Forth, they answered their height, weight, age, the number of years of experience in various types of martial-art plays on questionnaire forms and the width between right and left acromia and the width between right and left iliac horns of the subjects were measured using a measuring tape.

\section{Subjects}

Fifteen healthy males (17 to 61 years old) who have experienced Tankendo practice for more than one year were included in the study as subjects. The best expert among them won the first prize in several national Tankendo championship.

jump distance

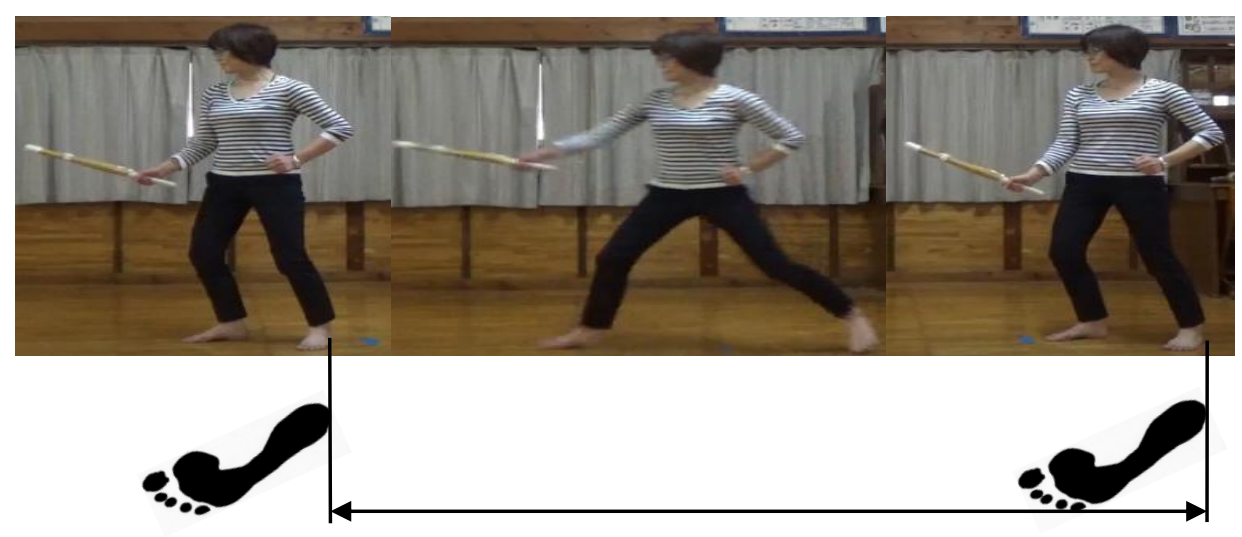

Figure 4: Jumping Over the Largest One of Subject's Favorite Distances

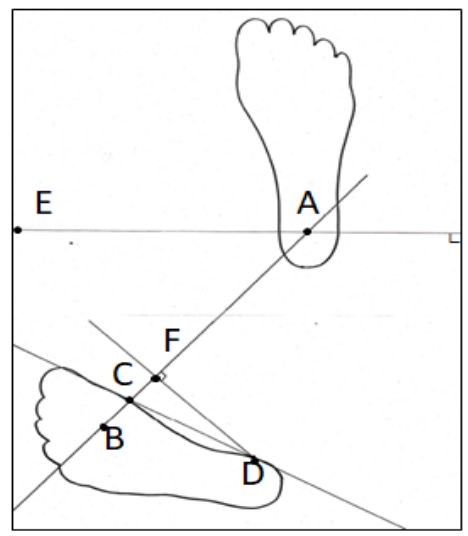

$$
\begin{aligned}
& d=\overline{A B} \\
& \theta 1=\angle E A B \\
& \theta 2=\angle C D F
\end{aligned}
$$

Figure 5: Kinematics of Foot Posture

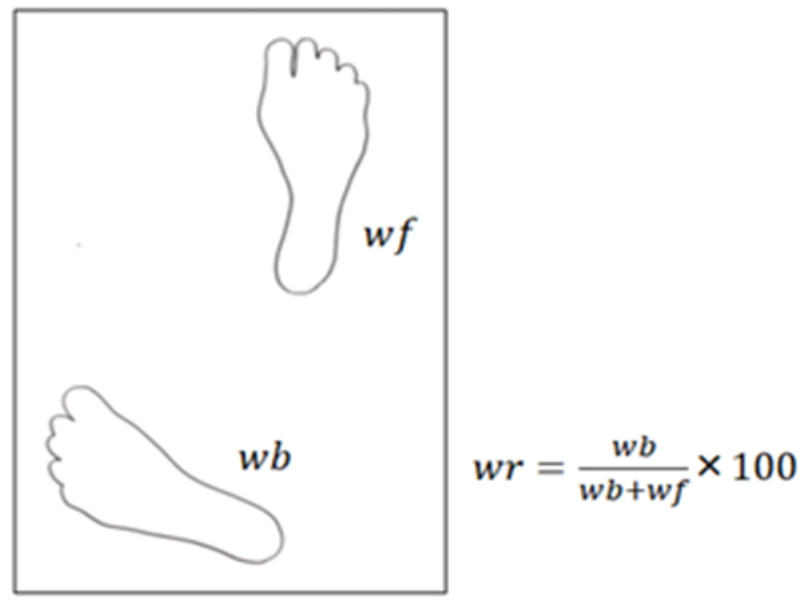

Figure 6: Weight Distribution 


\section{ANALYSIS}

\section{Digitizations}

The traced footprints were visually digitized by an experimenter. Five feature quantities, $d$ (separation between the right and left feet), $\theta 1$ (backward angle of the left foot), $\theta 2$ (opening angle of the left toe), wr (weight distribution of the left foot) and jumping distance, were digitized. It should be noted that d (separation between the right and left feet) was normalized by means of height. wr (ratio of weight distribution of the left foot) was assumed to be the ratio to the whole weight. Moreover, BMI values obtained from the questionnaire forms were additionally digitized. The procedure for digitizing the feature quantities of foot posture is illustrated in Fig. 5.

First, it was assumed that the right foot pointed A and the left foot was point B as the sites, which served as pivots when the right and left feet started to step. The point A was the central point of a line connecting the right and left projections of the right heel. The point $\mathrm{B}$ was the point 1/3 apart inside from a line connecting the first metatarsal bone head and the fifth metatarsal bone head of the left foot. In addition, it was assumed that the first metatarsal bone head was point $\mathrm{C}$ and the projection inside of the left heel bone was point $\mathrm{D}$.

For the first feature quantity, d (separation between the feet), the distance between the points A and B were obtained.

For the second feature quantity, $\theta 1$ (backward angle of the left foot), the degree of movement of the left foot toward the heel side was measured using $\angle \mathrm{EAB}$.

For the third feature quantity, $\theta 2$ (opening angle of left toes), the degree of the opening degree of the left toes was measured using $\angle \mathrm{CDF}$.

The fourth feature quantity, wr (weight distribution of the left foot), was obtained by dividing the whole weight (wb+wf) by the weight exerted on the left foot (wf) followed by multiplication by 100 as shown in Fig. 6 using $w r=w b /(w b+w f) \mathrm{x}$ 100.

For the fifth feature quantity, jump distance, the distance over which the left heel bone moved, namely the distance the positions before and after the jump, was obtained.

\section{Methods}

The correlation among four feature quantities $(\mathrm{d}, \theta 1, \theta 2$, wr) obtained from the footprint traced on the paper was found and a one-to-one correlation among the foot posture and other feature quantities, body constitution (height, weight, MBI, width between metatarsal bone head points, width between iliac bones), age and level of player's skill (age, number of years of experience in Tankendo, the number of years of experience in Jukendo, the number of years of experience in Kendo), and jump distance, 14 in total, was obtained.

\section{RESULT AND DISCUSSION}

The mean values for four feature quantities for foot posture and jump distance were $18.6 \pm 3.3 \mathrm{~cm}$ for $d$ (separation between the feet), $40.6 \pm 10.1^{\circ}$ for $\theta 1$ (backward angle of the left foot), $1.5 \pm 12.3^{\circ}$ for $\theta 2$ (opening angle of the left toes) and $49.2 \pm 5.9 \%$ for $w r$ (ratio of weight distribution exerted on the left foot). Moreover, the jump distance was $85 \pm 17.9 \mathrm{~cm}$.

The relationships between player's body constitution, age, level of skill in martial-art plays, jump distances at striking and thrusting, and four feature quantities for foot posture are shown in Fig.7. In the upper figure, the feature quantities, for which a clear correlation with a correlation coefficient $\geqq 0.60$ was observed, are shown and in the lower figure, the feature quantities, for which a correlation tendency $\geqq 0.40$ was observed are shown. A solid line indicates a positive correlation and a dotted line indicates a negative correlation (a).
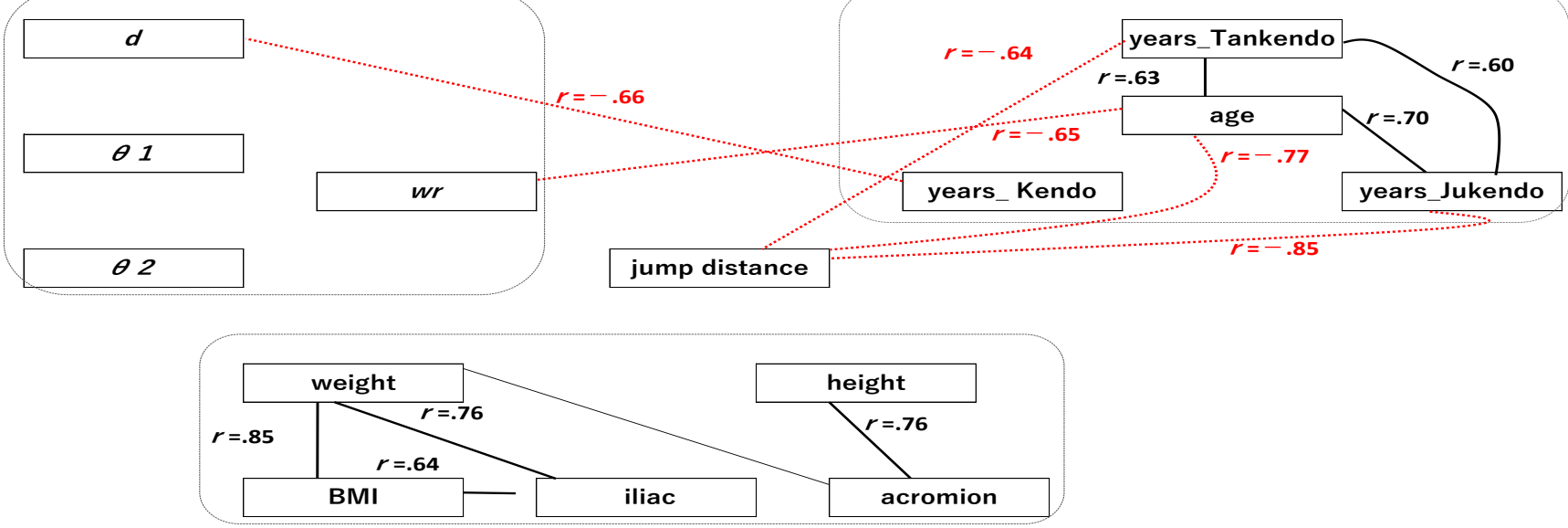
(b)

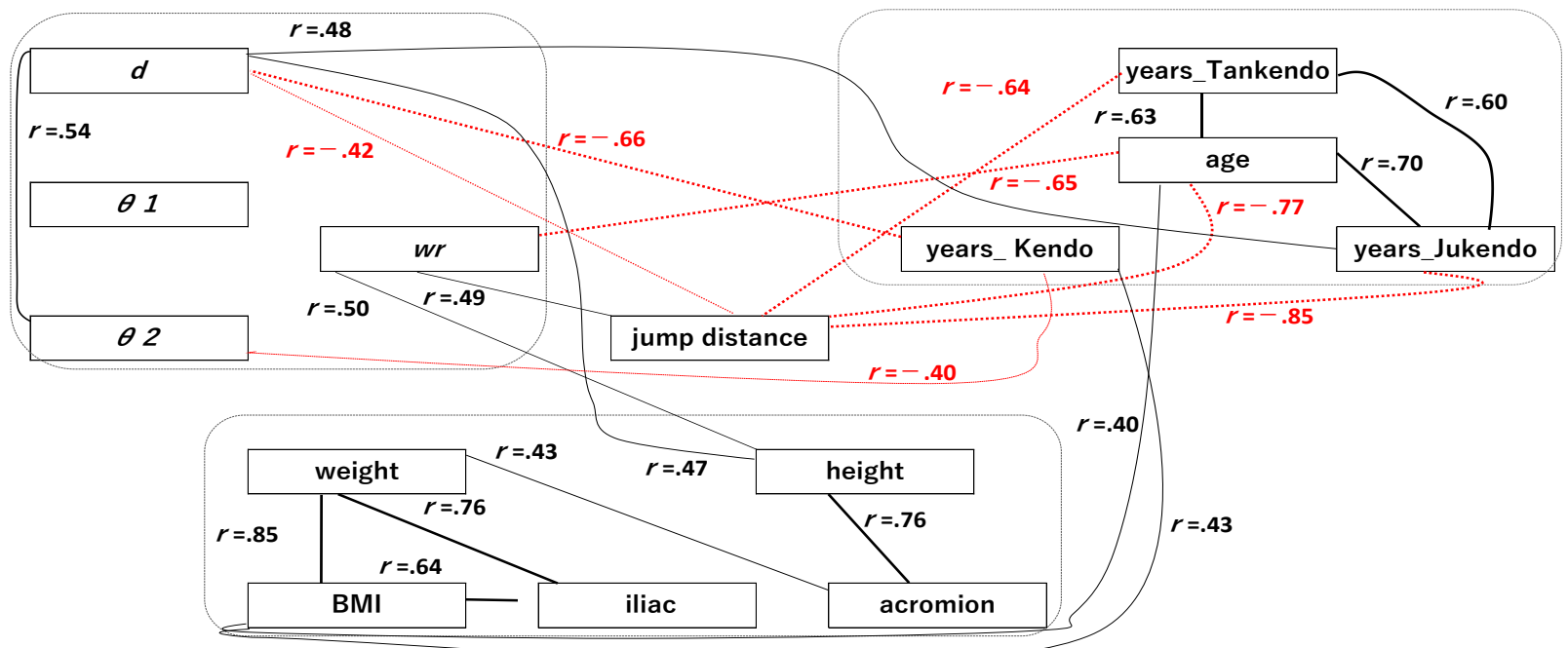

Figure 7: Correlation Coefficients between Feature Quantities

In the upper figure, the feature quantities, for which a clear correlation with a correlation coefficient $\geqq 0.60$ was observed, are shown and in the lower figure, the feature quantities, for which a correlation tendency $\geqq 0.40$ was observed are shown.

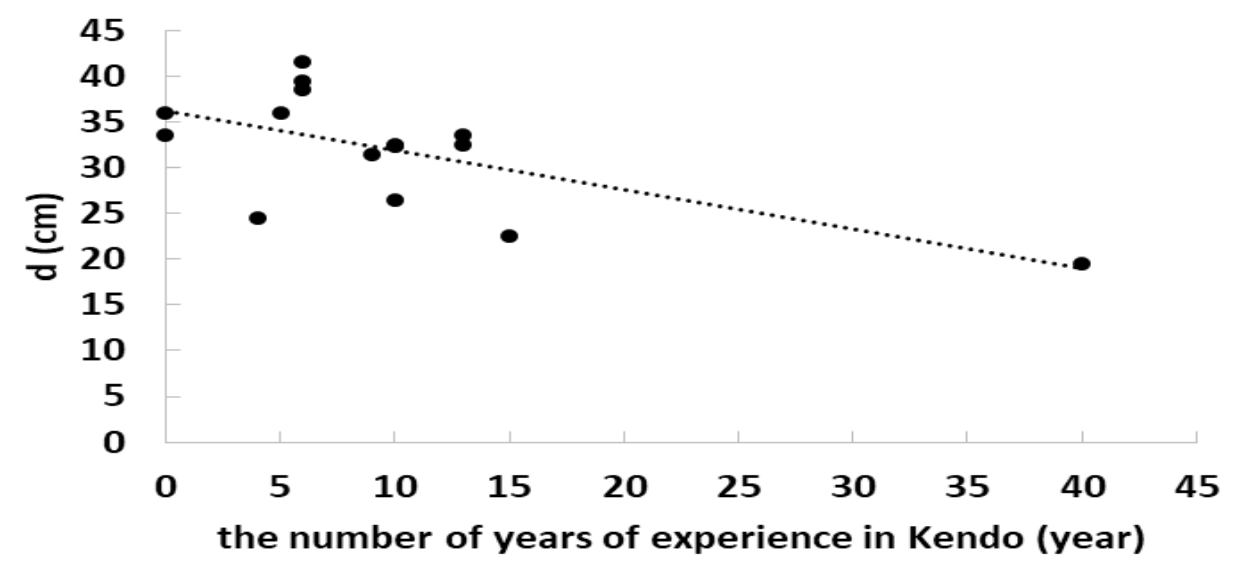

Figure 8: Experience in Kendo and Separation between the Feet

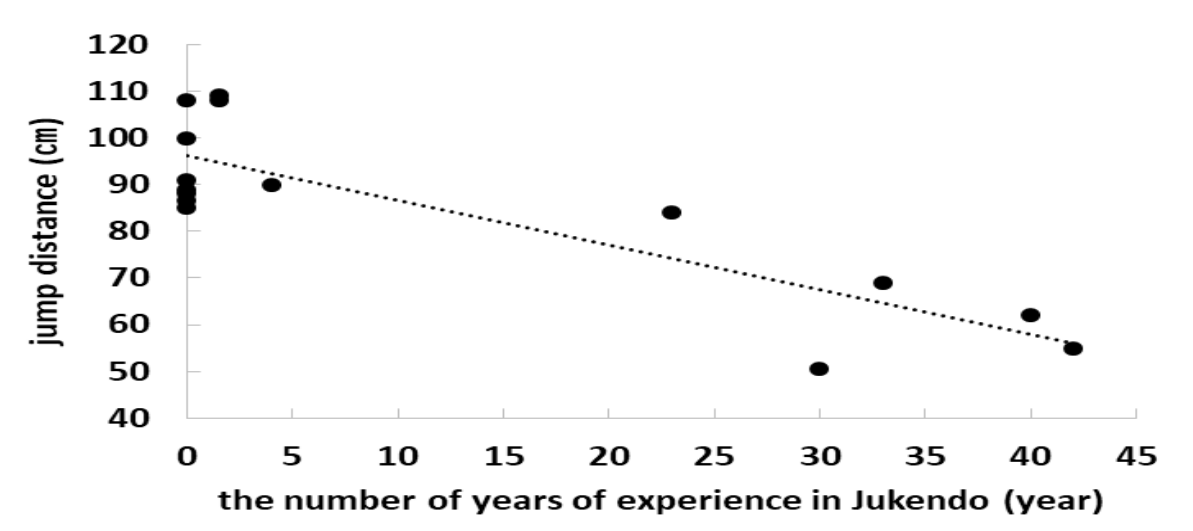

Figure 9: Experience in Jukendo and Jump Distance 


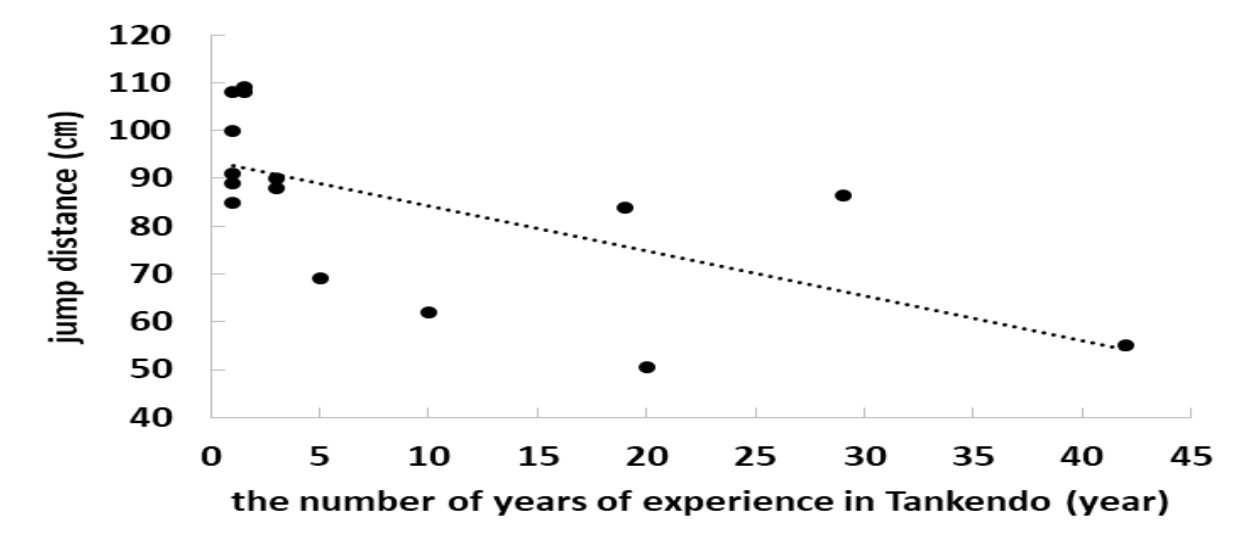

Figure 10: Experience in Tankendo and Jump Distance

The strength of the relationship between the items is shown by means of the thickness of the line. With a stronger correlation coefficient, the solid lines and dotted lines become thicker.

First, for the body constitution, with larger height, the width between the metatarsal bones becomes wider $(r=0.76)$, with larger weight, the BMI value becomes higher $(r=0.85)$, and the width between iliac bones also becomes wider $(r=0.76)$.

Second, looking at age and the number of years of experience in martial-art plays, with elder age, the level of skill becomes higher in Tankendo $(r=0.63)$ and Jukendo $(r=0.77)$ but no correlation was observed between age and the level of skill in Kendo $(r=-0.06)$.

Third, the level of skill in Tankendo had a positive correlation with the level of skill in Jukendo $(r=0.60)$.

No clear correlation $\geqq 0.60$ was observed among four feature quantities for foot posture.

Fourth, focusing on the relationship among the attributes, body constitution/age and levels of skill in martial-art plays, foot posture, and jump distance, a negative correlation was observed between the number of years of experience in Kendo and $\mathrm{d}$ (separation between the feet) $(r=-0.66)$ in Kendo.

In Jukendo, a negative correlation was observed between the number of years of experience in Jukendo and jump distance $(r=-0.85)$.

In Tankendo, similarly to Jukendo, a negative correlation was observed between the number of years of experience in Tankendo and jump distance $(r=-0.64)$.

Moreover, the result of single regression analysis assuming that the number of years of experience in Kendo was shown along the $\mathrm{X}$-axis and $d$ (separation between the feet) was along the $\mathrm{Y}$-axis using the pre-normalization data is shown in Fig.8. As known from the figure, the relationship of $y=-0.4311+36.245$ was observed between separation between the feet, $\mathrm{y}$ and the number of years of experience, $\mathrm{x}$ in Kendo.

Next, the result of single regression analysis assuming that the number of years of experience in Jukendo was shown along the $\mathrm{X}$-axis and jump distance was along $\mathrm{Y}$-axis, using pre-normalization data is shown in Fig.9. As known from the figure, the relationship of $y=-0.952 x+96.107$ was observed between jump distance, $y$ and the number of years of experience, $x$ in Jukendo.

Furthermore, the result of single regression analysis assuming that the number of years of experience in Tankendo was shown along the $\mathrm{X}$-axis and jump distance was along $\mathrm{Y}$-axis, using pre-normalization data is shown in Fig.10. In Tankendo, the relationship of $y=-0.94 x+93.70$ was observed between jump distance, $x$ and the number of years of experience, y. In short, the beginners tend to jump over $94 \mathrm{~cm}$ on average and as the number of years of experience increased by one year, the jump distance reduced by $1 \mathrm{~cm}$.

The results from a series of analyses suggested that the players tended to attack the opponent after approaching closely him/her rather than making an attack by one step from a distant place in Tankendo and Jukendo, both focusing on thrusting. In contrast, in Kendo focusing on striking, the tendency that the player with a higher level of skill attacked the opponent with the width between the feet narrowed.

It has been reported that in offense and defense in Kendo, skilled persons often "take quick actions of offense and defense at a distant spatial timing", while non-skilled persons often "take slow actions of offense and defense at a near spatial timing" (Yamamoto, 2013). In the future, a discussion taking a temporal factor related to the offense and defense at a temporal timing into account in addition to the inter-personal distance from the opponent is necessary and expected. 


\section{SUMMARY}

Tankendo is the martial-art play similar to Kendo, originated from Japan. It is largely different from Kendo in that the players use a relatively short $(53 \mathrm{~cm}$ in length) and light $(250 \mathrm{~g}$ in weight) bamboo sword. It focuses on close fighting. Since a short and light sword eliminate the need for the armed force and body strength and victory or defeat is determined by quick motions and ability to determine, women and elder persons also may enjoy this type of play.

A comparison is made among the techniques of domestic and overseas other "martial-art games using a sword". In Kendo mainly using striking techniques to attack the opponent, a player takes such a posture that his/her upper body faces straight to the opponent and both the feet face horizontally to the direction of movement with the right foot in the front. On the other hand, in fencing mainly using thrusting, a player turns the directions of his/her upper body and left foot by 90 degrees relative to the direction of movement. Moreover, similarly, in Jukendo, a player faces his/her upper body takes a posture almost at a right angle to the direction of movement to thrust the opponent with an oblique stance. However, in Jukendo, a player places his/her left foot in the front and his/her right foot at a distance separating horizontally on the outside so that both feet appear as if they indicated 2:00 on the clock face. In addition, in Tankendo using both the striking and thrusting techniques, some players take the foot posture similar to that in Kendo while others take like that in fencing. This study made a correlation analysis among the feature quantities to examine what types of factors determine the foot posture.

The experimental method is described below:

An A1-size paper (594 x $841 \mathrm{~mm}$ ) is attached on a flat wooden floor. First, the subjects with bare feet were requested to take his/her favorite foot posture at "a temporal and spatial timing of striking an "opponent" on that paper. In front of the subject, the opponent made face the subject with a short bamboo sword. Here a temporal and spatial timing of attacking the opponent means an inter-personal distance where a player may strike the opponent by one-stepping forward while may avoid striking by the opponent by one-stepping backward. Next the subjects traced the outlines of their feet on the paper with a fine felt tip pen. Moreover, in order to determine whether the heels were separated from the floor, a thin clear film was used to verify the position where the clear film might be inserted into the deepest point from the three directions: right, left and back sides.

Second, the subjects removed their feet from the paper. Two weight scales were put on the positions where the feet were placed. The subjects stood on the scales with the same standing posture to measure the weights exerted on both the feet.

Third, the subjects moved to the position slightly apart from the weight scales and paper and reproduce their foot posture. Then, they jumped over the largest one of their favorite distances seen in the game with body balance kept and the distances were recorded.

Fourth, they answered their height, weight, age, the number of years of experience in various types of martial-art plays on questionnaire forms and the width between right and left acromia and the width between right and left iliac horns of the subjects were measured using a measuring tape.

Fifteen healthy males (17 to 61 years old, the number of years of experience in Tankendo play), who have experienced in Tankendo practice for more than one year, were included voluntarily in this study. The best expert won the first prizes in the National Tankendo Championship several times.

The analysis was conducted as described below. First, the experimenter visually digitized the traced footprint. The digitized feature quantities included d (separation between the feet), $\theta 1$ (backward angle of the left foot), $\theta 2$ (angle of left toes), wr (ratio of weight distribution exerted on the left foot) and jump distance. It should be noted that $\mathrm{d}$ (separation between the feet) was normalized by means of height. Second, the BMI values were calculated in addition to numerical data obtained from the data on the questionnaire forms. The correlation was obtained among the four feature quantities obtained from the traced footprint and one-to-one correlation was also obtained among foot posture and other feature quantities, player's level of skill, body constitution, jump distance at striking and thrusting, etc. 14 in total.

The result showed that a strong negative correlation was observed between the level of skill and jump distance in Tankendo and Jukendo. On the other hand, in Kendo, a strong negative correlation was observed between the level of skill and separation between the feet. The result from a series of analyses suggested that in Tankendo and Jukendo focusing on thrusting, players tended to approach closely the opponent to attack rather than attack from a distant place by one-stepping while in Kendo focusing on striking, the player with a higher level of skill narrowed the separation between the feet when attacking the opponent.

\section{ACKNOWLEDGMENT}

The study was conducted partially under the financial support of Grand-in-Aid for Scientific Research by MEXT (NEXT/JSPS KAKENHI Grant Number 16K12199). Moreover, the study was conducted under approval by the Ethics Committee, Ibaraki Prefectural University of Health Sciences Approval number 775). 


\section{REFERENCES}

1. Aloofy, T. A., Al-Ansary, L., Mokhlis, L. G., Khalil, N. K., Alsamh, N. H. A., Faden, N. A., \& Borai, S. M. (2017). Public knowledge and practice of sore throat management among visitors of primary care clinic in Riyadh, Saudi Arabia. Journal of Advances in Health and Medical Sciences, 3(2), 1-8. https://doi.org/10.20474/jahms3.1.1

2. Hoshino, K., \& Cheng, C. (2016, November). Development of a swordsmanship machine enabling the inner and outer muscles to be safely trained while having fun. In Proceedings of the 3rd International Conference on Biomedical and Bioinformatics Engineering (pp. 59-62). ACM. https://doi.org/10.1145/3022702.3022711

3. http://www.geocities.jp/themusasi2g/gorin/g403.html/ (accessed 1 Octo-ber 2017)

4. http://www.kendo-progress.com/ (accessed 1 October 2017)

5. Kubo. T., Kizuka. T., Saitoh. M., Mutoh. K., Koda. K., \& Satoh. N. (2001). Effects of change of the distance between both feet of Chudan-no-Kamae on iEMG and force output of the lower limb muscle. Research Journal of Budo, 33(3), 12-25.

6. Tatsumi. N., \& Hattori. K. (1991). Lateral standing posture and body weight distribution during the standard ready position in Kendo. Research Journal of Budo, 23(3), 63-68.

7. Yamamoto, Y., Yokoyama, K., Okumura, M., Kijima, A., Kadota, K., \& Gohara, K. (2013). Joint action syntax in Japanese martial arts. Plos One, 8(9), e72436. https://doi.org/10.1371/journal.pone.0072436

8. Liu, H., \& Dong, X. (2016). Influence of social relations on knowledge conflicts-An empirical study from Chinese universities. Journal of Administrative and Business Studies, 2(1), 8-18. https://doi.org/10.20474/jabs2.1.2

9. Nuchso, N., Tuntivivat, S., \& Klayklueng, P. (2016). The effect of learning organization and servant leadership on child-centered teaching behavior with the mediating role of knowledge sharing in education of Chanthaburi Diocese schools. International Journal of Humanities, Arts and Social Sciences, 2(5), 181-188. https://doi.org/10.20469/ijhss.2.20004-5

10. Alahoul, M. H. M., Azizan, N., \& Alwi, N. H. (2016). Factors that affect the use of Malaysian e-learning websites by visually impaired users in the transfer of Islamic knowledge. Journal of Advanced Research in Social Sciences and Humanities, 1(1), 30-40. https://doi.org/10.26500/JARSSH-01-2016-0104

11. El-Ghalayini, Y. (2016). The effects of high performance work system on employee attitudes: A study of international organization. Journal of Administrative and Business Studies, 2(5), $248-263$. https://doi.org/10.20474/jabs-2.5.5

12. Siti Fatimah, A. Z., Norhafizah, M. S., Noryanti, M., Rozieana, K., \& Hassan, R. G. (2015). A study of students' performance in calculus and their attitudes toward the course using tripartite model. International Journal of Humanities, Arts and Social Sciences, 1(1), 30-35. https://doi.org/10.20469/IJHSS.20005

13. Gatpandan, M. P., \& Ambat, S. C. (2017). Implementing knowledge discovery in enhancing university student services portfolio management in higher education institutions, Journal of Advanced Research in Social Sciences and Humanities, 2(4), 211-220. https://doi.org/10.26500/JARSSH-02-2017-0306

14. HoshinoLab. (2015). Tankendo: close quarter combat with short bamboo sword. https://www.youtube.com/watch?v=XX8LMPKHmJk/ (accessed 1 October 2017).

15. Shimanoe, S., \& Hoshino, K. (2019). A wearable imaging system for recording the game tactics in martial arts using swords. Proceedings of IEEE 1st Global Conference on Life Sciences and Technologies (LifeTech 2019), 139-140.

16. Hoshino, K. (2018). The Method for Analyzing the Motions of Martial Arts. Patent Application No. 2018-27366, Filling date: Feb 19, 2018. (in Japanese)

17. Northey, J. M,. Cherbuin, N,. Pumpa, K. L., Smee, D. J., \& Rattray, B. (2018). Exercise interventions for cognitive function in adults older than 50: a systematic review with meta-analysis. British Journal of Sports Medicine, 52 (3), 154-160. https://doi.org/10.1136/bjsports-2016-096587

18. HoshinoLab. (2019). Tankendo All Japan 2019, the first round. https://youtu.be/L99S-UfS2V4/ (accessed 20 September 2019)

19. Turner, A., \& Harmenberg, J. (2017). Why fencers should bounce: a new method of movement to engage the stretch-shortening cycle. International Journal of Sports Science and Coaching, 13(3), 452-460. https://doi.org/10.1177/1747954117747133.

20. Koshida, S., \& Matsuda, T. (2013). Ankle and knee joint coordination in sagittal plane during kendo strike-thrust motion in healthy kendo athletes. Archives of Budo Science of Martial Arts and Extreme Sports, 9(2), $109-116$. https://doi.org/10.12659/AOB.889199

21. Hu, S., Jiang, M., Takaki, T., \& Ishii, I. (2018). Real-Time Monocular Three-Dimensional Motion Tracking Using a Multithread Active Vision System. J. Robot. Mechatron, 30(3), 453-466. https://doi.org/10.20965/jrm.2018.p0453

22. Purkayastha, S. N., Byrne, M. D., \& O'Malley, M. K. (2013). Human-Scale Motion Capture with an Accelerometer-Based Gaming Controller. J. Robot. Mechatron, 25(3), 458-465. https://doi.org/10.20965/jrm.2013.p0458 
23. Hoshino, K., Tamaki, E,. \& Tanimoto, T. (2007). Copycat hand - Robot hand imitating human motions at high speed and with high accuracy. Advanced Robotics, 21(15), $1743-1761$. https://doi.org/10.1163/156855307782506183

24. Sugimura, S., \& Hoshino, K. (2017). Wearable Hand Pose Estimation for Remote Control of a Robot on the Moon. J. Robot. Mechatron, 29(5), 829-837. https://doi.org/10.20965/jrm.2017.p0829

25. Miyajima, S., Tanaka, T., Miyata, N., Tada, M., Mochimaru, M,. \& Izumi, H. (2018). Feature Selection for Work Recognition and Working Motion Measurement. J. Robot. Mechatron, 30(5), $706-716$. https://doi.org/10.20965/jrm.2018.p0706

26. Hirose, S. (1998). Introduction of "Intelligent Sport". J. Robot. Mechatron , 10(1), $2-6$. https://doi.org/10.20965/jrm.1998.p0002

27. Maruyama, T., Tada, M., \& Toda, H. (2019). Riding Motion Capture System Using Inertial Measurement Units with Contact Constraints. Int. J. Automation Technol, 13(4), 506-516. https://doi.org/10.20965/ijat.2019.p0506 